\title{
Effect of allocated radiotherapy after breast surgeries over death risk in early breast cancer patients: a meta-analysis
}

\author{
V. ALBA-FERNÁNDEZ* and N. RUIZ-FUENTES \\ Department of Statistics and O.R., University of Jaén, Jaén, Spain
}

(Received 26 April 2006)

\begin{abstract}
Meta-analysis is a widely used tool to synthesize the results of a set of primary studies leading to more powerful conclusions than those provided by isolated ones. To evaluate the effect of allocated radiotherapy, types of breast surgery and other risk factors over death risk in early breast cancer patients, we carried out a meta-analysis using logistic regression attending to the dichotomous character of the outcome.

Our findings suggest that the type of breast surgery is an effect modifier of the risk factors over death in early breast cancer patients. The effect of allocated radiotherapy is associated with a less risk of death only for patients who had mastectomy plus axillary sampling. The effect of a systemic treatment is not associated with death risk for patients who had breast conservation, on the contrary it is associated for those patients with mastectomy alone and with mastectomy with axillary clearance.
\end{abstract}

Keywords: Meta-analysis; Early breast cancer; Risk factors; Logistic regression

AMS Classification: 62J05; 62P10

\section{Introduction}

Meta-analysis has become an essential tool for biomedical researchers in synthesizing the results of a set of studies about the same topic. Frequently, analysts think that it is enough to pool the results to obtain an overall average estimate of treatment effect. However, the analysts need to investigate the sources of variation of results across studies, in fact, a careful investigation of characteristics of study participants or design quality factors may provide clinically important results by indicating who might benefit more or less from a treatment or intervention.

There are several methods to investigate not only the presence of heterogeneity but also the potential sources of heterogeneity across studies. Within clinical trial research, to delimit the sources of heterogeneity we have to identify some study-level characteristics that are associated with the variation in the observed results. Subgroup analysis and meta-regression analysis are the most commonly used methods for doing this [1,2]. In our case, the interest focuses on finding sources of heterogeneity in dichotomous data by using logistic regression and study-level data.

*Corresponding author. Email: mvalba@ujaen.es

Computational and Mathematical Methods in Medicine

ISSN 1748-670X print/ISSN 1748-6718 online () 2006 Taylor \& Francis

http://www.tandf.co.uk/journals

DOI: $10.1080 / 10273660600777395$ 
In general, meta-analyses using individual patient data offer several advantages compared to those using study-level data, including the ability to use more appropriate statistical methods not always feasible when using study-level analysis. However, a meta-analysis using individual patient data is more expensive to conduct and requires more performing time. Furthermore, if potential explanatory variables have not been collected in primary studies, investigation of heterogeneity will be limited $[3,4]$.

The use and publication of meta-analysis as an evidence-based method has increased in the last decades. Focusing on the desirable quality-related methodological characteristics of a meta-analysis, we consider the suggestions of Stroup [5] who has identified methodological markers for quality of published meta-analyses at medical journals. Among these suggestions we take special care in a complete justification of the research, accompanied by the selection of an appropriate statistical methodology to pool data that allows the reader to replicate it.

In this paper, our aim is to combine systematically the results of a set of previous studies in order to arrive at conclusions about the effect of allocated radiotherapy and the type of breast surgery, among others, over the risk of death in early breast cancer patients. The results can be useful for many quantitatively-oriented medical researchers indicating who might benefit more or less from the applied treatments and suggesting the direction for future experiments and research.

To achieve our aim, we organize the paper as follows: the next section includes a concise account of the statistical method we have used, we also describe the data set and how the fitting procedure was performed. Section 3 gives a detailed description of the results which rise from the analysis. There we analyze the effect of those significant factors over the response variable and we also analyze the results under a clinical approach. In section 4 we discuss our results and make a comparison with other reported meta-analysis showing advantages and deficiencies of both methods.

\section{Method}

Logistic regression has become the standard method of multivariate analysis when describing the relationship between a discrete response variable, taking on two or more possible values, and one or more explanatory variables [6,7]. It is a model-building technique that, except for its own assumptions, follows most the principles and methods used in linear regression. The wide application of such techniques sometimes leads to a routine use of them without a rigorous and proper description of the procedure and with an unappropriated validation of its assumptions and results. These facts are associated with a misspecification of the model and may affect the decision making through the model results $[8,9]$.

The goal is to find the best parsimonious fitting but also a biologically reasonable model relating the response and the explanatory variables. This approach is quite useful for examining the effect of a treatment or intervention and other study-level characteristics over a response variable, so that it has a wide range of application in fields like epidemiology or medicine, in fact there are several terms that come from research in health sciences.

\subsection{Description of data}

Data have been collected from the paper "Favourable and unfavourable effects on long-term survival of radiotherapy for early breast cancer: an overview of the randomized trials" which was carried out by EBCTCG [10]. The data come from forty randomized trials that began 
before 1990 comparing radiotherapy plus other treatments (including breast surgery) versus the same other treatments without radiotherapy. These trials involved 18,592 (9830 deaths) women; they are classified under a stratification criterion attending to the type of breast surgery as follows: five trials involving 5125(3125) patients with mastectomy alone, six trials involving 3901(2106) patients with mastectomy with axillary sampling, 23 trials involving 6379(3585) patients with mastectomy with axillary clearance and six trials involving 4177(1022) patients with breast conservation with axillary clearance.

Let us describe the data: the response variable $Y$ is coded as 1 if a patient died and coded as 0 otherwise. The risk factor $X$ is coded as 1 for patients with allocated radiotherapy, and 0 for patients in the control group (i.e. without allocated radiotherapy). $G$ denotes the type of breast surgery, it is codified to identify four categories, the group-level aggregation leads to the value 1 for those trials with mastectomy alone, the value 2 for those trials with mastectomy with axillary sampling, 3 for mastectomy with axillary clearance, and 4 for breast conservation with axillary clearance, respectively. $C$ is coded to identify the presence or not of systemic therapy (whether it was chemotherapy or tamoxifen: data not shown), then $C$ is coded as 1 for those trials with systemic therapy and coded as 0 otherwise. Finally, we consider the primary studies as stratum and they are represented by $Z$.

Since the reported outcomes are dichotomous, binary logistic regression seems to be an appropriate technique to pool the data in order to evaluate the effect of the factors. We have also considered some study design factors and significant interactions between factors that may be clinically meaningful causes of heterogeneity.

\subsection{Model}

Let $\pi$ be the probability of death $(Y=1)$ at a combination of categories of the covariates and

$$
\operatorname{logit}=\ln \left(\frac{\pi}{1-\pi}\right) .
$$

The odds of death $\pi /(1-\pi)$ will be denoted as $W$. The fitted logistic regression model establishes the relationship between the logit and the covariates as follows

$$
\begin{aligned}
\operatorname{logit}= & \beta_{0}+\beta_{1} X+\beta_{2} C+\beta_{3} G^{1}+\beta_{4} G^{2}+\beta_{5} G^{3}+\beta_{6} X \times G^{1}+\beta_{7} X \times G^{2}+\beta_{8} X \times G^{3} \\
& +\beta_{9} C \times G^{1}+\beta_{10} C \times G^{2}+\beta_{11} C \times G^{3}+\sum_{j=1}^{k-1} \beta_{1_{j+1}} Z^{j}
\end{aligned}
$$

where $G^{i}, Z^{j}$, for $i=1,2,3$ and $j=1, \ldots, 39$ are dummy variables.

To assess the fit of the logistic regression model we proceed with a goodness-of-fit test, the value of the Hosmer-Lemeshow statistic computed from the frequencies in table 1 is 1.260 , and the corresponding p-value computed from the chi-square distribution with 8 degrees of freedom is 0.996 , this indicates that the model seems to fit quite well. A comparison of the observed and expected frequencies in each cell in table 1 shows that the model fits within each decile.

\section{Results}

In this section we highlight some of the results from the model attending to the considered study-level characteristics. The primary studies were considered as stratum and the fitted 
Table 1. Observed and estimated expected frequencies to compute the Hosmer-Lemeshow statistic.

\begin{tabular}{llcccccccccc}
\hline & \multicolumn{10}{c}{ Deciles } \\
\cline { 3 - 12 } Exitus & Frequencies & 1 & 2 & 3 & 4 & 5 & 6 & 7 & 8 & 9 & 10 \\
\hline 1 & Observed & 344 & 492 & 763 & 893 & 1047 & 1209 & 1105 & 1095 & 1297 & 1593 \\
& Expected & 340.7 & 499.6 & 753.3 & 909.2 & 1036.2 & 1209.3 & 1104.9 & 1090.8 & 1297 & 1597 \\
0 & Observed & 1671 & 1214 & 1128 & 1069 & 905 & 871 & 710 & 688 & 784 & 704 \\
& Expected & 1674.3 & 1206.4 & 1137.7 & 1052.8 & 915.8 & 870.7 & 710.7 & 692.2 & 784 & 700 \\
\hline
\end{tabular}

model reveals that its contribution is significant. Table 2 shows in an ordered sequence the odds ratio to compare de $i$ th stratum with the last one (that is, with stratum $k=40$ ). These results allow a comparison between strata. We briefly comment on some of these results, we can say that strata 17, 39, 27, 31 and 16 are homogeneous with respect to the reference strata, $k=40$. The risk of death is less for those patients from strata $k=36$ than for those at the reference one, and it is higher for the remaining significant values, more than four times or than seven times for stratum $k=23$ and $k=6$, respectively.

We have an immediate comparison between breast surgeries, see table 3 . All the comparisons lead to significant differences excluding that between mastectomy alone and mastectomy with axillary clearance $(\mathrm{OR}=1.179, \mathrm{CI}(0.703-1.974))$. For the aforementioned groups the risk of death is more than twice than for the group of breast conservation with axillary clearance $(\mathrm{OR}=2.668, \mathrm{OR}=2.263)$, respectively. However, the risk of death in the group of mastectomy plus axillary sampling is more than twice if we compare with them $(\mathrm{OR}=2.0903, \mathrm{OR}=2.4644$, respectively).

Finally, let us remark the comparison concerning to mastectomy plus axillary sampling versus breast conservation with axillary clearance, it leads to a higher risk of death for the first group than for the second $(\mathrm{OR}=5.577, \mathrm{CI}(4.195-7.415))$.

Once the effect of the covariates has been analyzed we proceed to evaluate the effect of those significant interactions. Covariate $C$ is referred as systemic treatment, its effect is analyzed taking into account the group the patients come from because the interaction between Group $(G)$ and systemic treatment $(C)$ is significant $(p \approx 0$ ). In respect of $C$, it has been coded as 1 for those trials applying systemic therapy and coded as 0 otherwise, and the significance of the interaction term implies that it has to be interpreted for each category of Group $(G)$.

The interaction also implies that the results given about breast surgeries refer to the reference level of the covariate $C$, that is for patients with a systemic treatment. Let us analyze the most relevant results comparing breast surgeries for patients without systemic treatment $(C=0)$, see table 4 .

The results show that the risk of death for patients with mastectomy alone is lightly higher, but no significant, than for those who also had axillary clearance $(\mathrm{OR}=1.3513$, $\mathrm{CI}(0.78-2.34))$ whereas it is more than five times with respect to those patients who had

Table 2. Analysis of strata.

\begin{tabular}{llllllllllllllllll}
\hline Strata & 36 & 17 & 8 & 38 & 39 & 2 & 3 & 1 & 32 & 16 & $\ldots$ & 25 & $\ldots$ & 18 & 23 & 6 \\
$\mathrm{e}^{\beta}$ & 0.532 & 0.656 & 0.666 & 0.698 & 0.782 & 0.974 & 0.98 & 1.057 & 1.201 & 1.273 & $\ldots$ & 1.6 & $\ldots$ & 4.263 & 4.561 \\
N.S. & & $*$ & & & $*$ & $*$ & $*$ & $*$ & $*$ & $*$ & & & & & & &
\end{tabular}


Table 3. Comparison between breast surgeries.

\begin{tabular}{|c|c|c|c|c|}
\hline Comparison & & & OR & $95 \% \mathrm{CI}$ \\
\hline $\mathrm{M}^{\dagger}$ alone & vs & $\mathrm{M}$ with axillary clearance & 1.1790 & $(0.703,1.974)$ \\
\hline M with axillary sampling & & $\mathrm{M}$ alone & 2.0903 & $(1.188,3.683)$ \\
\hline M with axillary clearance & & $\mathrm{BC}^{\ddagger}$ with axillary clearance & 2.2630 & $(1.527,3.353)$ \\
\hline M with axillary sampling & & M with axillary clearance & 2.4644 & $(1.524,3.986)$ \\
\hline $\mathrm{M}$ alone & & $\mathrm{BC}$ with axillary clearance & 2.6680 & $(1.629,4.369)$ \\
\hline $\mathrm{M}$ with axillary sampling & & BC with axillary clearance & 5.5770 & $(4.195,7.415)$ \\
\hline
\end{tabular}

${ }^{\dagger}$ Mastectomy.

${ }^{\ddagger}$ Breast conservation.

mastectomy plus axillary sampling $(\mathrm{OR}=5.5067, \mathrm{CI}(3.053-9.9725))$ and for those with breast conservation with axillary clearance $(\mathrm{OR}=5.6508, \mathrm{CI}(4.322-7.391))$. In addition to this, it is worthwhile to remark that the risk of death for patients who had mastectomy with axillary clearance is more than four times than for those with mastectomy plus axillary sampling $(\mathrm{OR}=4.075, \mathrm{CI}(1.877-8.883))$, and than for those with breast conservation with axillary clearance $(\mathrm{OR}=4.1820, \mathrm{CI}(2.365-7.3996))$.

Breast surgeries also act as an effect modifier of the risk factor $(p=0.001)$, now we interpret some of the results shown in table 5 and referred to this interaction term. All groups have a higher risk of death than the group of breast conservation with axillary clearance. The risk of death for those patients who had mastectomy plus axillary sampling is seven times that for those with breast conservation with axillary clearance $(\mathrm{OR}=6.9545)$ and it is more than twice for those with mastectomy with axillary clearance $(\mathrm{OR}=2.0276)$ and for those with mastectomy alone $(\mathrm{OR}=2.5453)$.

In the following, we analyze the effect of the systemic treatment for each breast surgery separately. The results in table 6 allow us to conclude that for the fourth group, breast conservation with axillary clearance, the risk of death is the same irrespective of the patients were treated or not by a systemic treatment $(\mathrm{OR}=0.8354, \mathrm{CI}(0.606-1.152))$. For the first (mastectomy alone) and third (mastectomy with axillary clearance) groups, the risk of death is less if patients are treated with a systemic treatment $(\mathrm{OR}=0.3944$, $\mathrm{OR}=0.4521)$. Finally, for those patients who had mastectomy with axillary sampling, the risk of death is more than four times if they have been systemic treated $(\mathrm{OR}=4.5403$, $\mathrm{CI}(2.555-8.1))$.

Now let us analyze the effect of the allocated radiotherapy over each breast surgery, see table 7 . There is only a significant value for those patients who had mastectomy with axillary sampling, the risk of death is less for those who had allocated radiotherapy (OR $=0.7558$, $\mathrm{CI}(0.666-0.859))$. The remaining comparisons lead to homogeneous groups.

Table 4. Comparison between breast surgeries without systemic treatment.

\begin{tabular}{|c|c|c|c|c|}
\hline Comparison & & & OR & $95 \% \mathrm{CI}$ \\
\hline $\mathrm{M}$ alone & vs & $\mathrm{M}$ with axillary clearance & 1.3513 & $(0.784,2.34)$ \\
\hline M with axillary clearance & & $\mathrm{M}$ with axillary sampling & 4.0750 & $(1.877,8.883)$ \\
\hline $\mathrm{M}$ alone & & $\mathrm{M}$ with axillary sampling & 5.5067 & $(3.053,9.9725)$ \\
\hline M with axillary sampling & & $\mathrm{BC}$ with axillary clearance & 1.0262 & $(0.556,1.888)$ \\
\hline $\mathrm{M}$ with axillary clearance & & $\mathrm{BC}$ with axillary clearance & 4.1820 & $(2.365,7.3996)$ \\
\hline $\mathrm{M}$ alone & & BC with axillary clearance & 5.6508 & $(4.322,7.391)$ \\
\hline
\end{tabular}


Table 5. Comparison between breast surgeries without allocated radiotherapy.

\begin{tabular}{|c|c|c|c|c|}
\hline Comparison & & & OR & $95 \% \mathrm{CI}$ \\
\hline $\mathrm{M}$ alone & vs & $\mathrm{M}$ with axillary clearance & 1.2553 & $(0.748,2.1004)$ \\
\hline M with axillary sampling & & M with axillary clearance & 3.4298 & $(2.484,4.732)$ \\
\hline $\mathrm{M}$ alone & & M with axillary sampling & 0.3660 & $(0.2357,0.567)$ \\
\hline $\mathrm{M}$ with axillary sampling & & $\mathrm{BC}$ with axillary clearance & 6.9545 & $(5.069,9.534)$ \\
\hline $\mathrm{M}$ with axillary clearance & & BC with axillary clearance & 2.0276 & $(1.367,3.008)$ \\
\hline $\mathrm{M}$ alone & & $\mathrm{BC}$ with axillary clearance & 2.5453 & $(1.5503,4.169)$ \\
\hline
\end{tabular}

\section{Discussion}

After to present the most meaningful conclusions of our analysis, we briefly comment some features about the original analysis made by EBCTCG. In that paper, a standard metaanalysis based on the Peto's method has been performed. For each trial, the effect of allocated radiotherapy over the mortality rates was analyzed by considering a logranks statistic. Afterwards, all these statistics were added for an overall analysis. The statistical heterogeneity of these logrank was tested by means of the DerSimoniard-Laird test [11]. The $p$-value revealed homogeneity between all the studies.

After a stratified study according to the type of breast surgery, the heterogeneity analysis between the four subtotals revealed statistical differences, but this test cannot indicate in what sense or direction such difference occurs. No other stratification was done using the DerSimoniard-Laird test. Therefore, this type of analysis does not allow us to detect nor identify why the observed mortality rates vary. In fact, the results obtained through this analysis does not provide us enough explanation of the effect of factors on the mortality rates and their observed variation. We think that it is necessary to use a more sophisticated technique that may provide more accuracy in investigating the heterogeneity results and which also takes into account the original binary character of the data.

These facts motivated the use of binary logistic regression to provide clinically useful findings by indicating what kind of patients may obtain higher benefits from a particular treatment. Moreover, the fitted model allow us to explain almost ever the variation across study results, the percentage of deviance explained by the model was around $97 \%$.

Now, we point out in a brief way some of the most meaningfully results that can be drawn from the fitted model, the effect of allocated radiotherapy is associated with a less risk of death only for patients who had mastectomy with axillary sampling, this effect is not significant for the remaining groups.

The effect of a systemic treatment is not associated with death risk for patients who had breast conservation, its application has an increasing risk for those who had mastectomy with axillary sampling. However, for patients with mastectomy alone and with mastectomy with axillary clearance, the risk of death is reduced if they were systemic treated.

Table 6. Effect of systemic treatment for each breast surgery.

\begin{tabular}{lcl}
\hline Breast surgery & OR & \multicolumn{1}{c}{$95 \%$ CI } \\
\hline M alone & 0.3944 & $(0.2533,0.613)$ \\
M with axillary sampling & 4.5403 & $(2.555,8.1)$ \\
M with axillary clearance & 0.4521 & $(0.248,0.825)$ \\
BC with axillary clearance & 0.8354 & $(0.606,1.152)$ \\
\hline
\end{tabular}


Table 7. Effect of allocated radiotherapy for each breast surgery.

\begin{tabular}{lll}
\hline Breast surgery & OR & \multicolumn{1}{c}{$95 \%$ CI } \\
\hline M alone & 0.9879 & $(0.883,1.108)$ \\
M with axillary sampling & 0.7558 & $(0.666,0.859)$ \\
M with axillary clearance & 1.052 & $(0.95,1.166)$ \\
BC with axillary clearance & 0.9425 & $(0.816,1.088)$ \\
\hline
\end{tabular}

When we compare between breast surgeries we find opposite behaviour depending on treatments, for instance, the risk of death is higher for those patients who had mastectomy with axillary sampling than for those who had breast conservation with axillary clearance if patients were systemic treated, but if they were not systemic treated the risk of death is not associated with the type of intervention.

\section{References}

[1] Oxman, A.D. and Guyatt, G.H., 1992, A consumer's guide to subgroup analysis, Annals of Internal Medicine, 116, $78-84$.

[2] Thompson, S.G. and Higgins, J.P.T., 2002, How should meta-regression analyses be undertaken and interpreted, Statistics in Medicine, 21, 1559-1573.

[3] Berlin, J.A., Shmid, D.H., Szczeh, L.A. and Feldman, H.I., 2002, Individual patient versus group-level data meta-regression for the investigation of treatment effect modifiers: ecological bias rears its ugly head, Statistics in Medicine, 21, 371-387.

[4] Lambert, P.C., Sutton, A.J., Abrams, K.R. and Jone, D.R., 2002, A comparison of summary patient-level covariates in meta-regression with individual patient data meta-analysis, Journal of Clinical Epidemiology, 55, $86-94$.

[5] Stroup, D.F., Thacker, S.B., Olson, C.M., Glass, R.M. and Hutwagner, L., 2001, Characteristics of metaanalyses related to acceptance for publication in a medical journal, Journal of Clinical Epidemiology, 54, $655-660$.

[6] Hosmer, D.W. and Lemsehow, S., 1989, Applied Logistic Regression (New York: Willey).

[7] Kleinbaum, D.G., 1994, Logistic Regression: A Self-Learning Text (New York: Springer-Verlag).

[8] Bagley, S.C., Whitee, H. and Golomb, B.A., 2001, Logistic regression in the medical literature: Standards for use and reporting, with particular attention to one medical domain, Journal of Clinical Epidemiology, 54, 979-985.

[9] Bender, R. and Grouven, U., 1996, Logistic regression models used in medical research are poorly presented, British Medical Journal, 313, 628.

[10] EBCTCG, 2000, Favourable and unfavourable effects on long-term survival of radiotherapy for early breast cancer: an overview of the randomised trial, The Lancet, 355, 1757-1770.

[11] DerSimoniard, R. and Laird, N., 1986, Meta-analysis in clinical trials, Controlled Clinical Trials, 7, 177-188. 


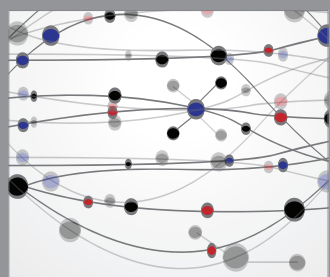

The Scientific World Journal
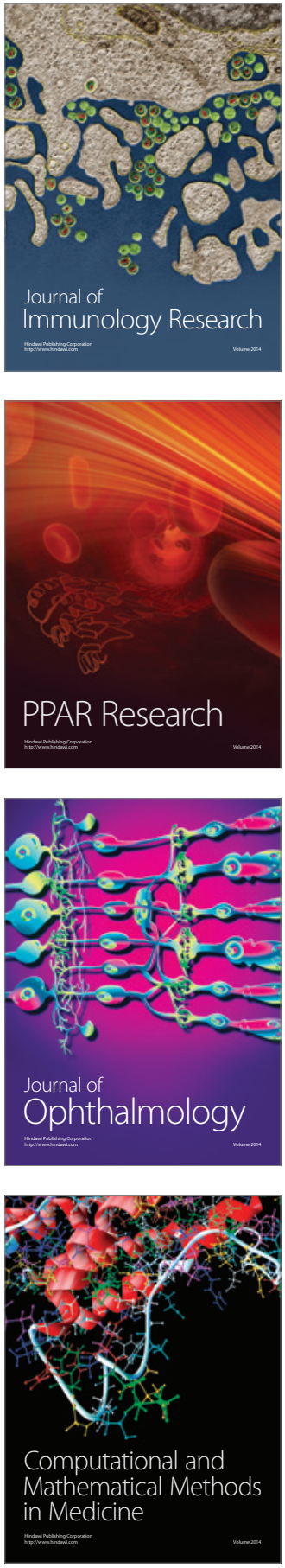

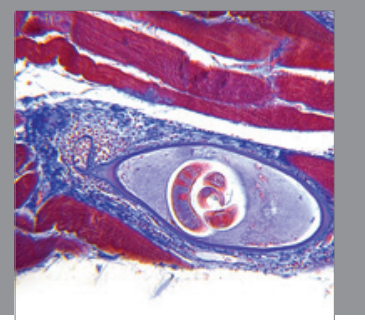

Gastroenterology

Research and Practice
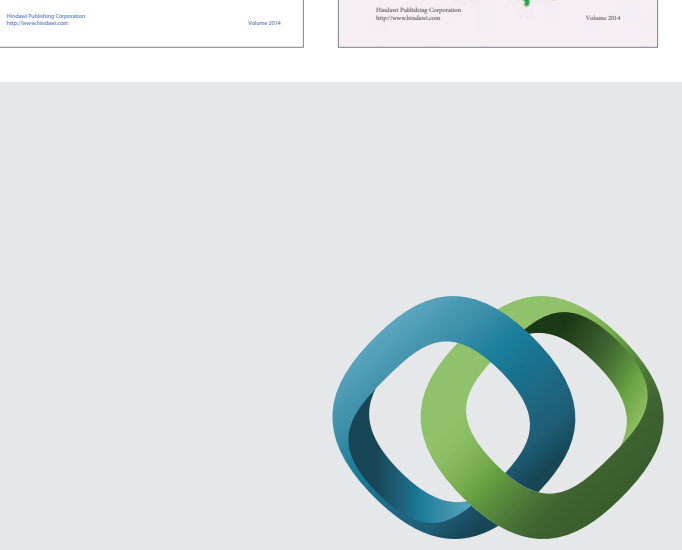

\section{Hindawi}

Submit your manuscripts at

http://www.hindawi.com
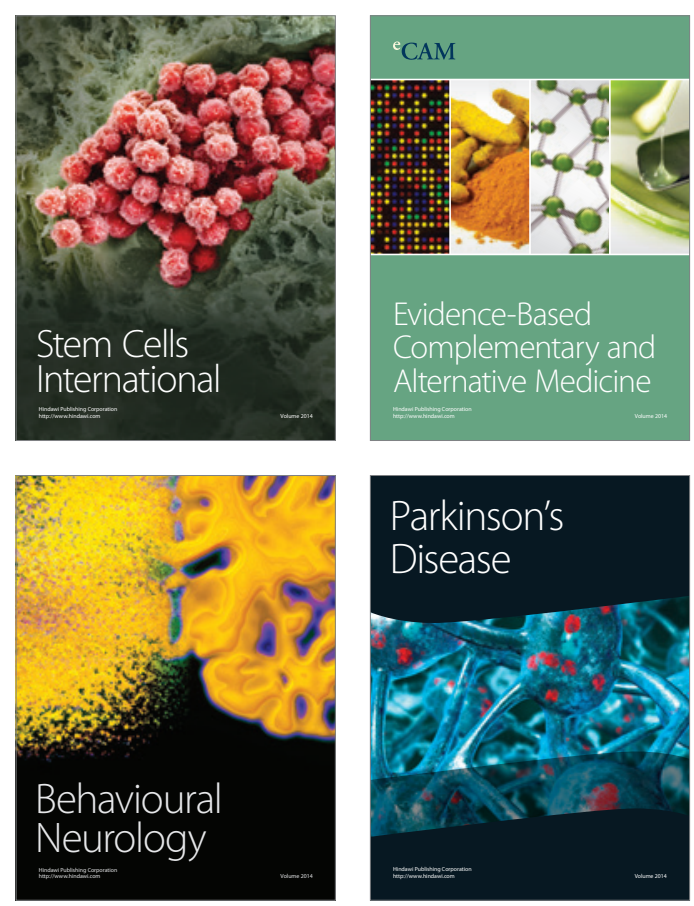

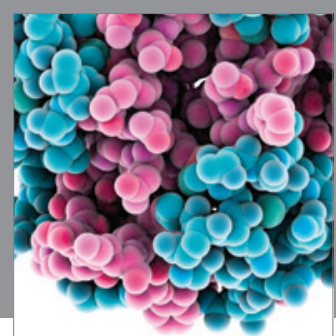

Journal of
Diabetes Research

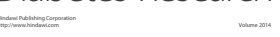

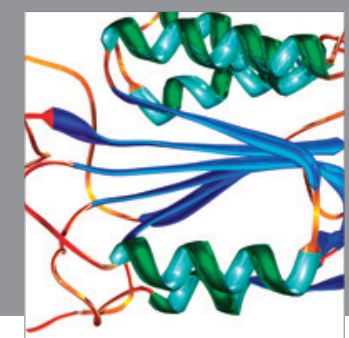

Disease Markers
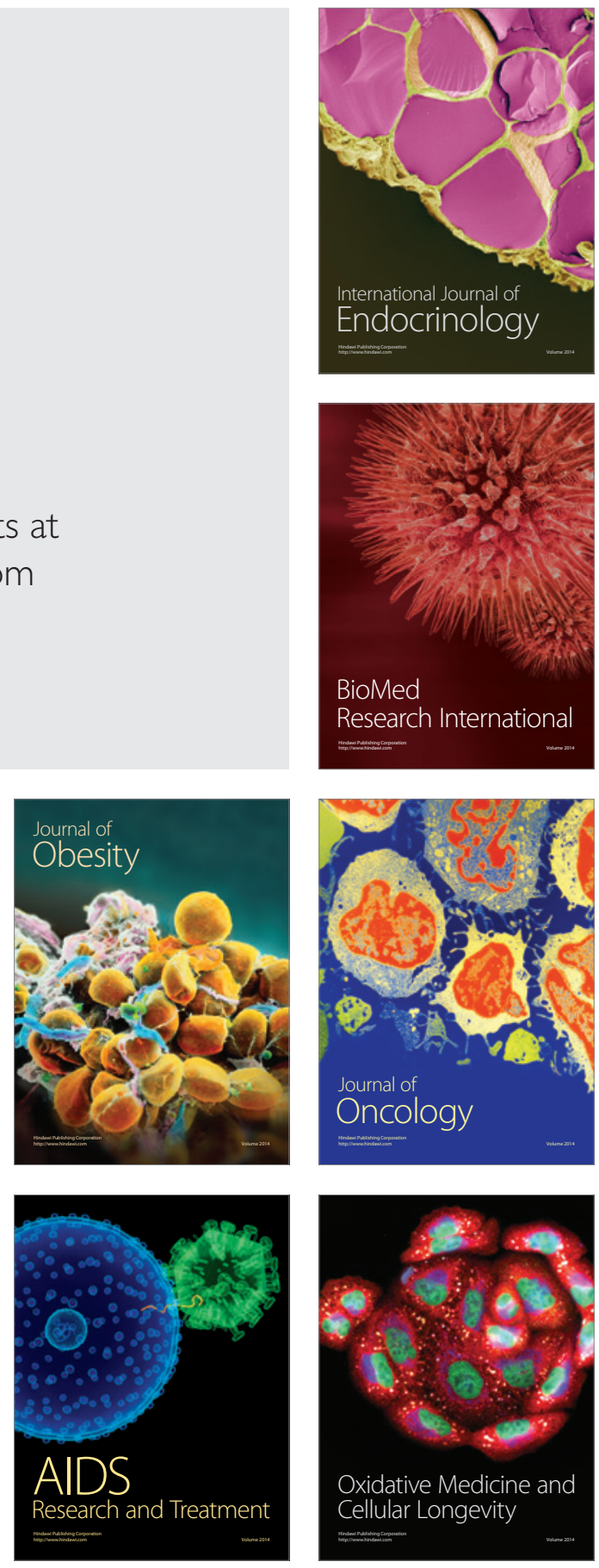